Short Paper

\title{
Zur Fruchtberostung 2018
}

\author{
About the russetting of apples in 2018 \\ Considerazioni sulla rugginosità delle mele nel 2018
}

\author{
Martin Thalheimer ${ }^{1}$ \\ ${ }^{1}$ Versuchszentrum Laimburg
}

\section{ABSTRACT}

Fruit russetting of apples originates from injuries of the fruit epidermis and the subsequent suberification of the damaged tissue. Fruit russetting can be caused by a variety of factors and their complex interactions.

Whereas some forms of fruit russetting, such as frost rings, can be unequivocally attributed to frost events, diffuse russett may be caused by a variety of factors and can therefore not be attributed to specific climatic events. Also in the case of the widespread fruit russetting observed in the apple growing area of South Tyrol in 2018 with its prevalently diffuse appearance it is therefore not possible to unequivocally determine the precise cause of the damage.

\section{KEYWORDS}

apples, russetting, fruit quality, climate

\section{CITE ARTICLE AS}

Thalheimer Martin (2019). About the russetting of apples in 2018.

Laimburg Journal 1/2019, DOI: 10.23796/LJ/2019.007.

\section{CORRESPONDING AUTHOR}

Martin Thalheimer

Laimburg 6, Pfatten, 39040 Auer (BZ), Italien

martin.thalheimer@laimburg.it +390471969652 


\section{EINLEITUNG}

Die Fruchtberostung von Äpfeln stellt eine bedeutende Ursache für die qualitative Abstufung der Früchte und daher einen empfindlichen finanziellen Wertverlust dar. Das Auftreten von Fruchtberostung bei Äpfeln ist auf eine Schädigung von Zellverbänden in der Fruchtepidermis zurückzuführen, welche zumeist in den frühen Phasen der Fruchtentwicklung auftritt [1]. Die für das menschliche Auge sichtbare Berostung entsteht durch die nachfolgende Verkorkung der Fruchtschale im Bereich der geschädigten Zellen.

Die möglichen Ursachen für das Entstehen von Fruchtberostung sind vielfältig. Dazu zählen die genetische Prädisposition einer Sorte oder eines Klons [2], sowie klimatische, chemische [1] [3] [4] [5] und biologische [2] [4] [6] [7] [8] Einflussfaktoren.

Zwischen den verschiedenen auslösenden Faktoren gibt es auch vielfältige Wechselbeziehungen, weshalb das Auftreten dieses Phänomens generell schwer vorhersehbar und rückwirkend auch nur sehr eingeschränkt erklärbar ist [1].

Zur Problematik der Fruchtberostung gibt es zahlreiche Fachpublikationen, welche im Laufe vieler Jahrzehnte veröffentlicht wurden. So erschien bereits 1938 eine detaillierte, mittels lichtmikroskopischer Untersuchungen erstellte Studie über die histologischen Veränderungen bei der Entstehung der Berostung [9], in der 2. Hälfte des vorigen Jahrhunderts kamen dann auch elektronenmikroskopische Untersuchungen hinzu [10].

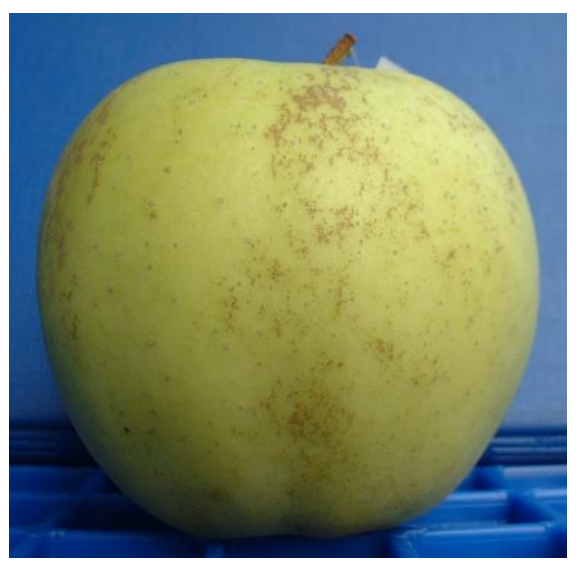

Abb. 1: Diffuse Berostung als Folge einer organischen Blattdüngung in der umittelbaren Nachblütezeit // Diffuse fruit russetting caused by the application of an organic foliar fertilizer in the post-bloom period.
Während die histologischen Veränderungen der Fruchtschale beim Phänomen der Berostung mittlerweile hinreichend beschrieben und erklärt sind, gibt es hinsichtlich der physiologischen Mechanismen und der auslösenden Umweltfaktoren weit weniger Klarheit.

Im Jahr 2018 trat bei verschiedenen Apfelsorten (z.B. Pinova, Golden Delicious, Scilate Envy ${ }^{\circledR}$, Scifresh $\mathrm{Jazz}^{\circledR}, \mathrm{CIVG} 198 \mathrm{Modi}^{\circledR}$ ) in weiten Teilen des Südtiroler Anbaugebiets in überdurchschnittlichem Ausmaß Fruchtberostung auf. Als möglicher auslösender Faktor wurden Kälteeinbrüche in der 2. Märzhälfte, also im Zeitraum der phänologischen Stadien von Knospenaufbruch bis Mausohrstadium vermutet. Die Berostung zeigte sich allerdings vorwiegend als diffuse Berostung (siehe Abb. 1) und nicht in der ansonsten für Frostschäden typischen Form von Frostringen (siehe Abb. 2) oder Frostzungen.

\section{DER EINFLUSS KLIMATISCHER FAKTOREN AUF DIE FRUCHT- BEROSTUNG}

Der klimatische Verlauf spielt eine unbestrittene Rolle beim Auftreten von Fruchtberostung. Besonders für den berostungskritischen Zeitraum in den Wochen um und nach der Blüte ist dieser Einfluss vielfach dokumentiert. Vor allem den Faktoren Wasser und Temperatur kommt in diesem Zusammenhang eine besondere Bedeutung zu. Allein schon die Präsenz von Wasser auf der Fruchtschale kann in diesem sensiblen Zeitraum zu Mikrorissen und in der Folge zu Berostung führen [11] [12]. Der schädigende Einfluss von Frost während oder unmittelbar vor der Blüte ist in der Praxis seit jeher bekannt und kommt - neben der völligen Schädigung der Blüte - insbesondere durch das Entstehen von Frostringen und Frostzungen zum sichtbaren Ausdruck. Die Mechanismen, welche zur Schädigung der Knospen oder Blüten führen, sind komplex und nicht allein durch die klimatischen Gegebenheiten erklärbar. Dem physiologischen Zustand der Knospen kommt eine besondere Bedeutung zu. Dieser hängt vorrangig von der Entwicklungsphase, dem Ernährungszustand und dem vorangehenden klimatischen Verlauf ab. Mit der fortschreitenden phänologischen Entwicklung der Blütenknospen kommt es generell zu einer Zunahme der Frostempfindlichkeit.

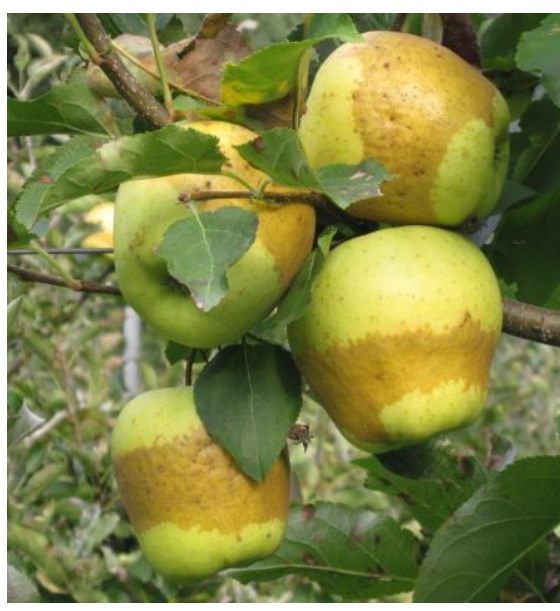

Abb. 2: Frostringe als Folge von Frühjahrsfrösten // Frost rings caused by late spring frost.

Die typischen Schadbilder, welche mit Frostereignissen im Zeitraum um und vor der Blüte zusammenhängen, sind neben der völligen Schädigung der Blüte das Auftreten von lokal abgegrenzten Bereichen von Fruchtschalenberostung, meistens in Form von Frostringen oder Frostzungen.

\section{ZUR PROBLEMATIK DER BEROS- TUNG IM JAHR 2018}

Angesichts des verbreiteten Auftretens von diffuser Fruchtberostung bei Äpfeln im Jahr 2018 wurde wiederholt die Vermutung geäußert, dass die Kälteeinbrüche vor oder bei Vegetationsbeginn als auslösender Faktor angesehen werden könnten. Sowohl Mitte als auch Ende Februar 2018 war es zu Temperatureinbrüchen mit Mindesttemperaturen deutlich unter dem Gefrierpunkt gekommen. Am Versuchszentrum Laimburg wurde am 15.02.2018 auf $50 \mathrm{~cm}$ über dem Boden eine Mindestemperatur von $-8.8^{\circ} \mathrm{C}$ und am 28.02.2018 von $-8.1{ }^{\circ} \mathrm{C}$ aufgezeichnet. $\mathrm{Zu}$ diesen Zeitpunkten hatte bei den Hauptsorten das Knospenschwellen noch nicht eingesetzt, mit Ausnahme der Sorte Cripps Pink, bei welcher bereits am 20.02.2018 das Knospenschwellen erkennbar war. Weitere Frostereignisse von bescheidenerem Ausmaß wurden im Laufe des Monats März verzeichnet, wobei als tiefster Messwert auf $50 \mathrm{~cm}$ Messhöhe am 22.03.2018 eine Temperatur von $-3.5{ }^{\circ} \mathrm{C}$ registriert wurde. Im Laufe des Monats April wurden keine Minusgrade mehr verzeichnet. Der Vegetationsbeginn entsprach 2018 weitgehend dem langjährigen Mittel. Am Versuchszentrum Laimburg erreichten die Hauptsorten die Vollblüte um Mitte April. 


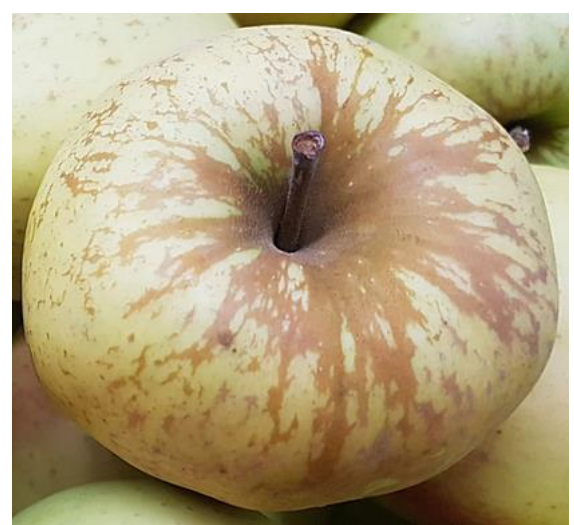

Abb. 3: Häufiges Erscheinungsbild der Fruchtberostung bei Golden Delicious im Jahr 2018 // Common appearance of fruit russetting on Golden Delicious in 2018.

\section{Foto: (C) Hansjörg Sachsalber, MIVOR}

Das verbreitete, starke Auftreten von Fruchtberostung ohne eindeutige Frostsymptomatik im Jahr 2018 stellt für den Südtiroler Obstbau ein ungewöhnliches Ereignis dar (siehe Abb. 3). Eine detaillierte Recherche in der Fachliteratur ergab keine Hinweise für das Auftreten von diffuser Berostung als Folge von Frostereignissen, während diese Art der Berostung sehr wohl als Folge chemischer, biologischer oder sonstiger Umwelteinflüsse in der Nachblütezeit dokumentiert ist. Besonders der Einsatz bestimmter Spritzprodukte in der berostungsempfindlichen Phase nach der Blüte kann diffuse Fruchtberostungen auslösen, wobei allerdings auch die jeweils vorherrschenden klimatischen Bedingungen eine sehr bedeutsame Rolle spielen.

Im Nordosten der USA kam es beispielsweise 2013 zu einem weit verbreiteten Auftreten von Fruchtberostung bei Äpfeln, wobei die Ausbringung von Fungizidmischungen als möglicher Auslöser in Betracht gezogen wurde. Daraufhin durchgeführte gezielte Untersuchungen bestätigten den möglichen Berostungseffekt von Kombinationen einiger, in der berostungsempfindlichen Zeit häufig eingesetzter Wirkstoffe [13].

\section{ZUSAMMENFASSUNG}

Die Fruchtberostung bei Äpfeln wird durch eine Schädigung der Fruchtepidermis verursacht und entsteht durch die Verkorkung der geschädigten Zellbereiche. Die möglichen Ursachen für die Schädigung der Fruchtoberfläche sind vielfältig und können komplexen Wechselwirkungen unterliegen.

Während einige Formen der Fruchtberostung (Frostringe, Frostzungen) eindeutig auf Frost als auslösenden Faktor zurückgeführt werden können, wird diffuse Berostung durch verschiedene Einflussfaktoren verursacht und kann folglich nicht eindeutig spezifischen klimatischen Ereignissen zugeordnet werden. Auch bei der im Jahr 2018 im Südtiroler Apfelanbau verbreitet aufgetretenen Fruchtberostung mit vorwiegend diffuser Ausprägung ist es demnach nicht möglich, eine eindeutige Ursache als auslösenden Faktor zu ermitteln.

\section{RIASSUNTO}

La rugginosità delle mele viene provocata dal danneggiamento dell'epidermide e dalla sua conseguente suberizzazione. Le cause di questo fenomeno sono molteplici e possono interagire in maniera complessa tra di loro.

Mentre alcune forme di rugginosità (placche, cinghiature) sono sintomi tipici di eventi di freddo, la rugginosità diffusa può essere causata da molteplici fattori e di conseguenza non può essere ricondotta ad eventi climatici ben precisi. Anche nel caso della rugginosità delle mele riscontrata nel 2018 in larga parte dell'area melicola dell'Alto Adige e che si è manifestata prevalentemente sotto forma diffusa, il fenomeno non è ricollegabile con certezza ad una causa precisa.

Das Fehlen von wissenschaftlich dokumentierten Zusammenhängen zwischen Kälteereignissen in der Vorblütezeit und diffuser Fruchtberostung erlaubt es natürlich auch nicht, im Umkehrschluss einen diesbezüglichen kausalen Zusammenhang kategorisch auszuschließen. Da es sich bei der Fruchtberostung, wie bereits erwähnt, um ein komplexes, mehrfaktorielles Phänomen handelt, gibt es immer wieder Situationen, bei denen die auslösenden Ursachen nicht eindeutig identifiziert werden können. Im Jahr 2010 trat beispielsweise in Deutschland in biologisch wirtschaftenden Betrieben bei der Sorte Jonagold eine verstärkte Fruchtberostung auf, für welche keine eindeutig nachvollziehbare Erklärung gefunden werden konnte [14]. 


\section{LITERATUR}

[1] Teviotdale B. L., Viveros M., Grant J. A. (1997). Apple russetting influenced by more than copper sprays. California Agriculture 51 (1), 11-14, DOI: 10.3733/ca.v051n01p11.

[2] Falginella L., Cipriani G., Monte, C. et al. (2015). A major QTL controlling apple skin russeting maps on the linkage group 12 of 'Renetta Grigia di Torriana'. BMC Plant Biology 15:150, DOI: 10.1186/s12870-015-05074.

[3] Nejatzadeh Barandoozi F., Talaie A. (2009). The effect of gibberellins on russeting in golden delicious apples. Journal of Horticulture and Forestry 1 (4), 61-64, Retrieved March 12,2019, from https://academicjournals.org/journal/JHF/article-full-textpdf/D310B054999.

[4] Gildemacher P., Heijne B., Silvestri M. et al. (2006). Interactions between yeasts, fungicides and apple fruit russeting. FEMS Yeast Research 6 (8), 1149-1156, DOI: 10.1111/j.1567-1364.2006.00109.x.

[5] Wang L., Li J., Gao J. et al. (2014). Inhibitory effect of chlorogenic acid on fruit russeting in 'Golden Delicious' apple. Scientia Horticulturae 178 (October), 14-22, DOI: 10.1016/j.scienta.2014.07.038.
[6] Gildemacher P., Heijne B., Houbraken J. et al. (2004). Can phyllosphere yeasts explain the effect of scab fungicides on russeting of Elstar apples? European Journal of Plant Pathology 110 (9), 929-937, DOI: 10.1007/s10658-004-8948-x.

[7] Heidenreich M. C. M., Corral Garcia M. R., Momol E. A., et al. (1997). Russet of apple fruit caused by Aureobasidium pullulans and Rhodotorula glutinis. Plant Disease 81 (4), 337-342, DOI: 10.1094/PDIS.1997.81.4.337.

[8] Easterbrook M. A., Fuller, M. M. (1986). Russeting of apples caused by apple rust mite Aculus schlechtendali (Acarina: Eriophyidae). Annals of Applied Biology 109 (1), 19, DOI: 10.1111/j.17447348.1986.tb03178.x.

[9] Verner L. (1938). Histology of apple fruit tissue in relation to cracking. Journal of Agricultural Research 57 (11), 813-824. Retrieved March 12, 2019 from https://naldc.nal.usda.gov/download/IND43969103/PDF.

[10] De Vries H. A. M. A. (1968). Development russeted of the structure of the apple skin. Acta Botanica Neerlandica 17 (5), 405-415, DOI: 10.1111/j.1438-8677.1968.tb00146.x.
[11] Rühmer T., Fellner M., Schafzahl, G. (2009). Versuchsbericht 2009-04 zur Schorfbekämpfung mit Pflanzenstärkungsmitteln. 1-7. Retrieved March 12, 2019 from http://www.agrar.steiermark.at/cms/dokumente/10935990 11730998/42bf57b2/200 9-04\%20Versuchsbericht\%20zur\%20Schorfbek\%C3\%A4mpfung\%20mit\%20Pflanzenst\%C3\%A4rkungsmitteln.pdf.

[12] Knoche M., Khanal B. P., Stopar M. (2011) Russeting and microcracking of 'Golden Delicious' apple fruit concomitantly decline due to gibberellin a4+7 application. Journal of the American Society for Horticultural Science 136 (3), 159-164, DOI: $10.21273 / J A S H S .136 .3 .159$.

[13] Villani S., Breth D., Cox K. (2015). Feeling the Burn! Chemical Injury on Apples Following Tank Mixtures of Captan, Single-site Fungicides, and Adjuvants. New York Fruit Quarterly 23 (1), 11-14. Retrieved March 12, 2019 from http://nyshs.org/wp-content/uploads/2015/03/NYFQ-Book-Spring-2015 compressed.pdf.

[14] Buchleither S., Späth S. (2010). Fruchtberostung 2010 bei Jonagold - das „Rätsel“ bleibt. Öko-Obstbau (3) 24-28. Retrieved March 12, 2019 from http://www.kob-bavendorf.de/arbeitsbereiche/oekologischer-obstbau/publikationen/resolveuid/ea59cd6e642e6c79d6f6a3dca3171 410.

\section{(c) $(1)$}

Dieses Werk ist lizenziert unter einer Creative Commons Namensnennung - Nicht kommerziell 4.0 International Lizenz.

Quest'opera è distribuita con Licenza Creative Commons Attribuzione - Non commerciale 4.0 Internazionale.

This work is licensed under a Creative Commons Attribution - NonCommercial 4.0 International License.

Für alle Abbildungen und Tabellen ohne Nennung des Urhebers gilt: (C) Versuchszentrum Laimburg.

Per tutte le immagini e tabelle senza menzione dell'artefice vale: (C) Centro di Sperimentazione Laimburg.

For all figures and tables without mention of the originator applies: (C) Laimburg Research Centre. 